\title{
Sistemas, modelos y decisiones. El impacto de la simulación en la administración de organizaciones complejas
}

Carlos Hernán González Campo 



\title{
Sistemas, modelos y decisiones
}

El impacto de la simulación en la administración de organizaciones complejas*

\author{
Carlos Hernán González Campo**
}

Recibido: Octubre 2006

Aceptado: Noviembre 2006
* Este artículo se constituye en un insumo para el proyecto de implementación del CUSE (Centro Universitario de Simulación Empresarial), proyecto que se inició en el segundo semestre del 2005, en la Facultad de Ciencias de la Administración de la Universidad del Valle.

** Magíster en Ciencias de la Organización. Profesor Auxiliar. Departamento de Administración y Organizaciones. Facultad de Ciencias de la Administración. Universidad del Valle. Cali-Colombia. 


\section{Introducción}

En nuestros días, las sociedades están constituidas por redes de organizaciones que funcionan como sistemas diferentes y complejos ${ }^{1}$ dentro de las que se pueden encontrar: las organizaciones industriales, las organizaciones comerciales de bienes y servicios, las organizaciones públicas, las organizaciones educativas, las organizaciones sin ánimo de lucro.

En nuestro análisis de mayor a menor complejidad, los niveles de construcción sistémica son la sociedad, los sub-sistemas son las organizaciones y las interacciones son los elementos relacionados. La evolución societal requicre en sus diferentes ctapas de procesos como la diferenciación social en la que intervienen transferencias de selección sin alterar el nivel de especialización de los elementos o subsistemas. Luhman en 1995, plantea esta complejidad desde el punto de vista de lo real, lo social y lo temporal, orientados a objetos, sujetos y al tiempo, respectivamente. El grado de importancia de esta diferenciación se demuestra con el grado de análisis sobre los diferentes tipos de sistemas y su complejidad.

Las diferentes ciencias tienen su método especifico, partiendo de sus principios, creados a partir de la experiencia o de las hipótesis, y demostrados siguiendo su método científico, una sucesión de operaciones que con- lleva a diferenciar los conocimientos, aplicados a situaciones reales y comprobados con la mayor exactitud posible, no sicmprc es exacto cl resultado, pero se pueden encontrar las mejores posibles alternativas de solución. (Popper, Adorno, Dahrendorf \& Habermans, 1969)

Desde esta perspectiva, la organización es un sistema compuesto de subsistemas, que recibe insumos como la energía, la información y los materiales del medio, los transforma y los regresa en forma de productos. Una organización es la integración de diferentes actividades humanas alrededor de distintas tecnologías, considerando que la organización interna está compucsta por varios subsistcmas principales, como: las metas y valores, técnico (conocimiento del proceso), psicosocial (individuos y grupos en interacción) y estructural (división del trabajo). El subsistema administrativo involucra todo lo que relaciona a la organización con su medio; establece los objetivos, desarrolla planes de integración, estrategia y operación, mediante el diseño de la estructura y el establecimiento de los procesos de control. (Johansen, 1992). Con relación a los sistemas artificiales, Katz y Kahn en 1979, plantean que por ser inventados por el hombre son imperfectos, constituidos por las actitudes, percepciones, creencias, motivaciones, hábitos y expectativas de los seres humanos, permitien-

\footnotetext{
Los sistemas complejos están constituidos por seres humanos inteligentes, seres humanos que son complejos por su naturaleza y su esencia. En términos de Morin (1994).
} 
do su análisis organizacional aplicando la Teoría General de Sistemas.

La Teoría General de Sistemas, el análisis organizacional basado $\mathrm{cn} \mathrm{cl}$ enfoque sistémico, la búsqueda de la explicación de algunos fenómenos, la solución de problemas y la toma de decisiones organizacionales, son elementos importantes para el desarrollo de los procesos de simulación.

Desde la mitad del siglo XX, cuando inicia el uso de la simulación en los procesos de enseñanza, se ha presentado un cambio pasando de un proceso de simplemente transferencia de información (un profesor que transmite sus conocimientos mediante clase magistral y artículos), a un proceso donde incluso sc reconoce la importancia de la enseñanza basada en la experiencia, termino que no fue nuevo para la época, ya para algunas escuelas de pensamiento como la aristotélica eran parte de su método, una de las metodologías mas utilizadas dentro de los procesos de enseñanza son los estudios de casos, principio básico en la constitución de simuladores (Brend, 1999).

En los modelos de simulación se plantea mostrar y descubrir el comportamiento de un sistema, se proponen hipótesis y teorías que intenten explicar el comportamiento observado, estas tcorías se pucden utilizar para predecir el comportamiento futuro del sistema, dependiendo de los cambios que se realicen sobre las variables y los parámetros, también pueden observar los posibles efectos en el funcionamiento del modelo y predicen lo que puede ocurrir en la realidad que intenta recrear el modelo de simulación planteado.

El impacto de la simulación en el desarrollo del individuo a nivel profesional y personal, se ha convertido en un clemento fundamental para su desempeño laboral, mejorando no solo su proceso al tomar decisiones, sino su integración con los otros elementos de la organización vista como un sistema, destrezas y competencias que el individuo adquiere en su proceso de formación con la utilización de los simuladores aplicados en diferentes situaciones.

Las competencias laborales están jugando un papel muy importante en los actuales procesos de estandarización de las organizaciones, para la formación basada en competencias técnicas y personales, es fundamental la utilización de simuladores en los procesos de aprendizaje, porque permiten no solo la transmisión de conocimientos teóricos sino que permite la generación de destrezas por medio de la practica en el entrono virtual recreado con la simulación.

\section{La complejidad de las organizaciones}

Es posible analizar cada instancia del funcionamiento de las organizaciones como sistemas. Un sistema es un conjunto de elementos organizados que se encuentran en intcracción, en búsqueda de un fin en común. Implicando características como: integridad, totalidad y unificación de partes para lograr un funcionamiento óptimo, los sistemas reciben entradas del entorno y contribuyen de diferente forma con salidas al mismo, el sistema se convierte 
en un procesador que transforman las entradas en salidas. Para la aproximación a este mundo de los sistemas en las organizaciones se plantca la Tcoría General de Sistemas (TGS), como un instrumento de análisis.

Estos sistemas se pueden clasificar de diferentes formas ${ }^{2}$ : según su naturaleza, entre: sistemas naturales (sistemas ecológicos), sistemas artificiales (los creados por el hombre) y sistemas sociales (relaciones entre el hombre y las máquinas). Según su relación con el medio ambiente se clasifican como: sistemas abiertos o cerrados (depende de su relación con el entorno), sistemas permanentes o temporales (dependiendo de la duración en el tiempo), sistemas cstables o no estables (variación interna de sus operaciones) y sistemas adaptativos o no adaptativos (cambia su funcionamiento según los cambios de su entorno). I os sistemas se pueden dividir en subsistemas y pertenecer a supersistemas donde se muestran la relación entre los diferentes elementos y niveles del sistema, dentro de los sistemas existen algunos conceptos importantes como son: a) las variables (entradas y salidas que tienen distintos valores), b) los parámetros del sistema (variables fijas), c) los componentes (existencia de subsistemas), d) los atributos de los componentes (pucden ser objetos o personas y sus propiedades y características), e) la estructura que identifica el conjunto de relaciones entre los objetos y atributos de los objetos de un sistema y su grado de funcionamiento juntos en búsqueda de objetivos en común; estas relaciones pucden ser: disfuncionales, parasitarias, simbióticas, sinergeticas y optimizadas, dentro de cada sistema se realiza el proceso de transformación de las entradas en salidas, las fronteras que se constituyen en el componente particular dentro de una jerarquía de sistemas, las interfases que es la región de contacto, la entropía que es el desgaste del sistema y por ultimo la homeostasis que es el avance hacia otro estado y dentro del mismo las características operacionales. Para la utilización de la Teoría General de Sistemas, en las organizaciones se deben tener en cuenta las condicioncs reales del problema y las necesidades de los encargados de tomar las decisiones, que son importantes en el diseño, construcción, producción, diagnostico y evaluación de los sistemas. Sistemas que se constituyen en el motor que realiza diversas actividades, en diferentes niveles de la organización con su respectiva especificidad, actividades desarrolladas teniendo en cuenta los agentes que constituyen la organización, las reglas o normas, los valores de la organización, su misión y visión, entre otros elementos importantes que definen a la organización, estos clementos de la organización como sistema interactúan en un mundo complejo en varias dimensiones de análisis. Es esta complejidad la que permite comprender aquellos fenó-

2 Partiendo de la clasificación realizada por Bertalanffy en 1984, solo se incluyen los tipos más relevantes. 
menos dentro de la organización y de su entorno, que dificultan el desarrollo de todas las actividades y procesos de la organización, no permitiendo cl cumplimiento de sus objetivos.

Es la complejidad social lo que diferencia a la sociedad de otros tipos de sistemas, pero a la vez es la que permite que por medio de la confianza (mecanismo de reducción de esta complejidad), se posibilite la implementación de proyecciones futuras sobre observaciones presentes de la realidad social con algún nivel de seguridad, sin garantizar altos grados de certeza, sino simplemente niveles de relación, en los términos planteados por Luhman en su construcción de su Teoría de la Sociedad.

Desde el punto de vista funcional, los medios de comunicación en una sociedad transmiten complejidad reducida. La función del poder como medio de comunicación es por tanto la de acabar con la discrepancia entre las selecciones de Alter y Ego, regulando así las contingencias a manera de catalizador que acelera o retarda este proceso. Esta acción catalizadora que actúa sobre la probabilidad sin cambiar el proceso, arroja como resultado una ganancia de tiempo importante en la construcción de sistemas complejos.

El poder como medio de comunicación simbólicamentc gencralizado, reduce la complejidad a través de la acción y no sólo a través de la experiencia por lo que su código supone la existencia de individuos en los extremos de la relación. El fenómeno del poder conceptuado por la Teoría de los Medios de Comunicación, diferen- cia el código y el proceso de comunicación eliminando el carácter de propiedad o facultad de una persona con respecto al poder que cjerce. La acción referida es aquella imputada al sistema y no a su entorno en la que las personas como sistemas toman selecciones-acciones, en definitiva toman decisiones. Los recursos disponibles para la sociedad se ven incrementados mediante la movilización, la formación de cadenas, la generalización y la especificación temática de los procesos de poder. Sin embargo la evolución social obedece a la contingencia y capacidad de diferenciación del poder, la política y la ley como base de la acción en cada etapa (Luhmann, 1995).

El poder es un factor universal necesario para la existencia de una sociedad, establecido en el mundo de la experiencia, involucrando niveles de confianza y reducciones de la complejidad que define a cada uno de los sistemas sociales. La complejidad se convierte en un resultado lógico en la Teoría General de Sistemas de propiedades de los sistemas como: la entropía, la sinergia y la evolución y crecimiento de los sistemas, incluyendo su imposibilidad de controlar los diferentes elementos que hacen parte de su entorno, los niveles de complcjidad están directamente relacionados con los tipos de sistemas o la equifinalidad otra de las propiedades que tienen los sistemas, donde con condiciones y caminos diferentes, existen varios modos y métodos para alcanzar un objetivo y así conseguir mejores resultados, in- 
clusive en las organizaciones complejas. (Bertalanffy, 1984).

Según Cornejo (2004), la complejidad como fenómeno, está asociada al tamaño de la organización, a las diferencias entre las organizaciones, a la competencia en el mercado, al no conocimiento, administración y gestión de toda la información posible ${ }^{3}$, a la no estandarización de los procesos y procedimientos dentro de una organización, al tipo de estructura de la organización, entre otras. Autores como Hall (1982), plantean: "La complejidad puede definirse como el grado de conocimiento requeridos para producir el resultado de un sistema", este análisis se reduce al nivel de la incertidumbre sobre cl funcionamicnto y en especial sobre los resultados que puede obtener la organización en el futuro ante las decisiones que se pueden tomar.

\section{EI método científico y los tipos de modelos}

En el análisis de las organizaciones y específicamente en la solución de problemas organizacionales, es fundamental, como herramienta el método científico, reconocido como el proceso organizado a seguir para obtener como resultado el conocimiento de una verdad dentro del planteamiento de una determinada escuela o disciplina científica, convirtiéndose en un conjunto de reglas que definen cada pro- ceso de investigación. En el método científico existen dos tipos de categorías: la teoría (hipótesis) y la practica (contrastación de la hipótesis), donde la ciencia es el resultado de la generación de conocimiento científico, proveniente de la investigación utilizando el método científico.

Existen dos tipos de métodos científicos, el deductivo, que intenta demostrar la verdad mediante la lógica pura, partiendo de las premisas planteadas sobre el problema a analizar, y el inductivo, que intenta crear leyes partiendo de la observación de los hechos en la realidad, con la generalización del comportamiento observado. El método científico es utilizado para la gencración de nuevos conocimientos científicos o para la comprobación de conocimientos ya existentes, se fundamenta en la recopilación, organización, en ocasiones la síntesis y al final el análisis de los datos obtenidos en el proceso de investigación.

Descartes en 1974 planteó, "Entiendo por método, reglas ciertas y fáciles, gracias a las cuales quien las observe exactamente no tomará nunca lo falso por verdadero, y llegará, sin gastar inútilmente esfuerzo alguno de su espíritu, sino aumentando siempre, gradualmente, su ciencia, al verdadero conocimicnto de todo aquello de que sea capaz".

Algunas disciplinas o escuelas de pensamiento, utilizan dentro de su mé-

3 Proceso por el cual aparece la incertidumbre como una propiedad de la racionalidad limitada planteada por Herbert Simon en 1972, uno de los principales autores de la Teoría de la Toma de Decisiones. 
todo científico los modelos para corroborar una hipótesis, los modelos son una representación de un objeto, sistema o idca de la rcalidad, guardando sus propiedades y características propias, que constituyen su esencia y lo diferencian de los otros entes similares. En la mayoría de los casos los modelos se utilizan como una herramienta para entender un sistema y experimentar reacciones ante cambios o variaciones del entorno o de sus variables.

Los modelos en su mayoría intentan ser una copia de la realidad, diferenciándose en el tamaño, material $\mathrm{u}$ otra característica sin perjudicar o variar lo que fundamenta su existencia en la realidad, en algunos casos puede scr una abstracción, incluyendo en su representación algunas características fundamentales del objeto o sistema. Teniendo como funciones: la de comparar (en el presente) o la de predecir (en el futuro), o en algunos casos las dos funciones al mismo tiempo. En su estructura básica, un modelo puede ser representado de forma matemática, por medio de la relaciónfunción entre sus variables y parámetros, o se puede expresar formalmente, por medio de símbolos que representan sus componentes (partes de un conjunto que forman el sistema), sus variables y cl proceso lógico de su funcionamiento. Estas variables que hacen parte de los modelos, pueden ser: 1) Exógenas (entradas que son originadas por causas externas al sistema) estadísticamente estas variables se procesan como independientes, o 2) Endógenas (son producidas dentro del sistema que resultan de las interacciones internas), las variables endógenas pueden ser de estado (muestran las condiciones iniciales del sistema), o de salida (son aquellas variables que resultan del sistema). I os parámetros son constantes susceptibles de manipulación por parte del operador del modelo, que puede asignarle diferentes valores sin ningún tipo de explicación adicional en algunos casos, en otros casos los valores de las constantes pueden depender de información interna o externa del modelo. Los modelos tienen por no ser exactamente la realidad, algunas limitaciones que se representan en los posibles limites de algunas de sus variables, limites que pueden aparecer de forma endógena o de forma exógena en el modelo.

Es muy importante tener en cuenta que dentro de los modelos existen relaciones funcionales que se forman del comportamiento de los parámetros dentro de uno o más componentes de un sistema, estas relaciones pueden ser determinísticas (donde una salida del proceso es determinada por una entrada), o estocásticas (donde el proceso tiene una salida indefinida), relaciones que se pucden representar por ecuaciones matemáticas generadas del análisis matemático y comprobadas en algunos casos por análisis estadísticos en forma de probabilidades o regresiones lineales. 


\section{La teoría general de sistemas y análisis organizacional}

Partiendo desde el punto donde se reconoce a la organización como un sistema ${ }^{4}$, se puede analizar su comportamiento con la Teoría General del Sistema, y en especial conocer el proceso de aprendizaje de la organización, el qué, cómo, dónde y de quién, aprenden las organizaciones, es un problema que puede ser estudiado desde una visión sistémica. La TGS, parte del problema de la definición sistema y entorno, esa línea divisora que tanto en la naturaleza como en las organizaciones o en la vida cotidiana del hombre, delimita cl sistema de su entorno, para ser reconocido como una totalidad, el sistema debe con sus propias operaciones funcionar como conjunto de forma sistémica, de lo contrario no dejarían de ser solo elementos aislados, y debe ser analizado desde esta perspectiva en la generación de sinergia, característica propia de los sistemas. Luhmann en 1997, describe la Teoría de los Sistemas Sociales, centrándose en la Teoría de la Comunicación como un componente característico de estos sistemas, especialmente en la transferencia de la información entre los clementos del mismo siste- ma, define que la transferencia de información entre sistemas o con su entorno, no es posible si hablamos de sistemas ccrrados, confirmando que cl hombre en algunos sistemas sociales, como por ejemplo las empresas comerciales siendo el cliente, no es parte del sistema sino que hace parte del entorno. Claramente esta Teoría de los Sistemas Sociales planteada por Luhmann, no parte de lo social sino de lo operacional del sistema, del como funciona una sociedad como procesos internos de un sistema. Del anterior argumento se basan la mayoría de las atribuciones que se le dan al hombre como excluido del sistema, mucho se ha discutido al respecto y desde diferentes puntos de vista, esto pucde ser cierto en el mundo tradicional ${ }^{5}$, pero por ejemplo en el nuevo mundo de los negocios electrónicos ${ }^{6}$, la comunicación entre los elementos del sistema se transfiere en forma de bits o de datos, por medios que se constituyen de redes de comunicación y de computo, compuestas por componentes físicos, lógicos y que por medio de protocolos de comunicación logran la transferencia de la información de un lugar a otro, almacenada y procesada en un sistema de información, en este caso todo el proceso y funcionamiento de la organización sc basa cn información,

4 Termino definido por Bertalanffy en 1986, desde la biología con la explicación del funcionamiento de la célula, que se aplica a varias disciplinas.

5 Entendiendo como mundo tradicional lo no virtual. En Negroponte(1995) el mundo de los átomos y no de los Bits.

6 Negocios electrónicos que se relacionan con temas como el E-Business, E-Commerce, E-Marketing, E-Logistic, E-Goverment, E-Trade, E-Learning, entre otros. 
el hombre en este modelo es representado por todas las características y preferencias de él mismo, datos recolectados en cada visita y cada transacción realizada por medio de un frontend tipo pagina Web o cualquier tipo de transacción por medios electrónicos donde sea plenamente identificado; incluso en el mundo tradicional, por medio de encuestas, degustaciones calificadas, tarjetas de cliente fiel o entrevistas personales, también se obtiene información del cliente, entonces es imposible excluir al hombre del sistema ya que no actúa como vinculo con otros sistemas, sino que es fundamental para que el mismo funcione, se desarrolle y alcance sus objetivos. Sin las preferencias del clicntc registradas, no seria posible construir un modelo lógico para su existencia y funcionamiento, de este tipo de negocios basados en información. Fstos nuevos sistemas sociales, son sistemas autopoieticos en términos de Luhmann, porque con su característica de estar constituido por datos, la transferencia entre los elementos de información hace que se construyan estructuras y elementos, que varíen para desarrollar y ofrecer productos personalizados para cada comprador de una forma masificada para todos los posibles compradores en el mercado de Internet o en los mercados tradicionales.

Conceptos aplicados de igual forma a todas las organizaciones, incluyendo diferentes teorías de los sistemas organizacionales que se basan también en la Teoría de la Comunicación y especialmente de la Teoría de la Acción, constituyéndose en el motor del funcionamiento de la organización, en este punto adquiere más importancia la premisa que para que se ejecute una acción es necesario una decisión(Luhmann,1997). Los elementos que constituyen un sistema organizacional son agentes tomadores de decisiones para actuar en el proceso del sistema. Lo virtual no es la realidad pcro es lo actual; para Ross (1981), Aristóteles, define la materia en cuatro capas como un referente al cambio; materia local, la materia para la alteración, la materia para el cambio del tamaño, la materia para nacer y perecer, este cambio se realiza en un tiempo y espacio especifico, en la virtualidad no podemos hablar de la materia, pero si hay una acción que genera un efecto sobre un fenómeno que posiblemente si puede ser real, una compra de un articulo por medio de Internet genera una cadena de procesos reales ${ }^{7}$, el aprendizaje virtual genera una construcción y apropiación

7 Cuando un cliente compra un producto en Internet con una tarjeta de crédito, una cadena de procesos se inician: Primero, la empresa proveedora solicita la autorización a la tarjeta de crédito del cliente para asegurarse de que la tarjeta es válida y que el cliente tiene disponibilidad para la compra. La tarjeta del cliente envía un número de autorización a la empresa o a su Website y la orden es ahora capturada por su empresa. El producto se envía después, y la empresa proveedora informa a la tarjeta la cuenta en donde deberá depositar los valores de la compra mientras que estos serán 
de conocimiento que es utilizado para la solución de problemas reales de forma autónoma inmediata de la organización, la comunicación intcractiva genera la satisfacción de la necesidad de compartir información, son acciones donde la materia no evoluciona o cambia en un espacio o un tiempo especifico para los que intervienen en el intercambio de información, la materia pierde algunas de sus propiedades que la convierten en virtual. La información se convierte en el ADN de las organizaciones de nuestro días, son los Bits una especie de átomos que construyen la organización, estos bits se transmiten de un lugar a otro por medio de las redes y operan todas las funciones del sistema, convirtićndose en un nuevo tipo de organizaciones basadas en información. (Negroponte, 1995)

Fn la teoría de I uhman, el sistema evoluciona debido a determinados mecanismos: la variación, la selección y la estabilización. El sujeto de la evolución es el sistema, cambian las formas en que el sistema se diferencia y los rasgos propios del sistema. La evolución es el progreso de la complejidad y la diferenciación, en los términos de Luhman. La mayor diferenciación sistémica supone una mayor estabilidad y capacidad del sistema para mantencrse estable en entornos que cada vez son más complejos. Evolucionan las formas de diferenciación, las formas concretas de la sociedad y la evolución de las formas sociales, la evolución es un proceso autorreferente en el sistema de diferencias y selecciones que crea sus propias condiciones y se detcrmina por medio de la diferenciación dentro de las propiedades de los sistemas y sus componentes. Para Morgan en 1998, en un sistema su comportamiento autorregulado depende de los procesos de intercambio de la información, posible por las propiedades de retroacción o realimentación negativa, principios que fundamentan los desarrollos de ciencias como la cibernética y que permiten la posibilidad que tienen los sistemas (en esta caso las organizaciones) de auto-organizarse por medio de sus interacción y su auto-aprendizaje. Las organizaciones aprenden internamente con el intercambio de información entre sus elementos o componentes y aprenden como sistemas por medio de la retroalimentación de información al procesar las entradas y salidas con la interacción con su entorno, entorno que puede ser el mercado competitivo. (Úbeda \& Llopis , 2002)

La posibilidad que tienen las organizaciones de ser analizadas como sistemas, y desde este punto de vista, sus propiedades, incluyendo la posibilidad de auto-organizarse y autoaprender, es lo que permite que por medio de un método científico, sc construyan modelos aproximados de la realidad, y de ellos se desarrollen procesos de simulación y simuladores, que utilizados en los procesos de aprendi-

debitados de la cuenta del cliente. En este momento, la tarjeta emite honorarios requeridos y la transacción es completada. 
zaje y experimentación, apoyen la toma de decisiones y la solución de problemas organizacionales.

\section{Orígenes de la simulación}

Dentro de los primeros autores interesados en la reforma de la teoría y de las practicas educativas que se convierten en bases para la simulación se encuentra, John Dewey que en 1949, en su planteamiento lógico y filosófico era de cambio permanente, adaptándose a las necesidades y a las circunstancias concretas, en varias de sus obras realiza un debate sobre exceso de teoría, en el análisis de los problemas y resolución de conflictos, con la poca utilización de la practica en la obtención de un resultado, debate que inicia la reforma de los métodos de enseñanza de la época, según Burch (1969), en 1957, se realiza la primera simulación en el campo de la organizaciones para resolver un problema gerencial, en la década siguiente se realizaron más de 100 simulaciones en diferentes campos organizacionales, en el año de 1962, se realiza por parte del profesor James McKenney, el primer estudio formal sobre el impacto de la simulación en la enseñanza en un proceso de producción, en un estudio realizado por Craig Eric Schncier en 1977, quc cxponc la existencia de mas simuladores que en su mayoría son nuevos desarrollos en el área administrativa, que cumplen con los estándares de acreditación propuestos por la AACSB (American Association of Collegiatc Schools of Business), en la siguiente década, se presenta un mayor desarrollo de la complejidad de los problemas que analizan los simuladores, incluyen ejercicios militares complejos, con múltiples decisiones y múltiples decisores entre sus áreas de trabajo, en 1996, dentro de un estudio realizado por Faria en 1990, se encuentran más de 18.000 instituciones entre Universidades, centros de capacitación y empresas, que utilizaban los simuladores en sus diferentes procesos de entrenamiento, el Instituto Tecnológico de Monterrey, fue la primera Universidad de habla hispana, cn utilizar los simuladores en el desarrollo de sus programas académicos en la década de los años sesenta en su Maestría en Administración, manteniéndose en la actualidad como institución líder en la utilización y desarrollo de simuladores en la región, en su Centro de Simulación Empresarial (CESIEM) ${ }^{8}$.

Autores como Lee en 1999, afirman que las simulaciones se clasifican en tres tipos básicos: simulaciones de presentación, simulaciones de práctica, y simulaciones híbridas. Las simulaciones de presentación se utilizan en los procesos de formación para ofrecer la posibilidad de comunicar nuevo conocimiento $^{9}$, las simulaciones de

8 http://www.ccm.itesm.mx/centro/

$9 \quad$ En esta dirección se pueden consultar varias simulaciones de este tipo. De carácter administrativo en http://www.gerentevirtual.com , aplicados a la ciencia en general en http://www.sciencesimulations.com 
práctica, se desarrolla en la practica de la teoría vista en clase magistral, por medio del simulador el estudiante utiliza la tcoría aprendida, sin la tcoría no pueden llegar a la practica, deben conocer previamente algunos elementos teóricos para trabajar con los simuladores, dentro de este tipo se encuentran la mayoría de simuladores comerciales que existen en la actualidad como: Markstrat (Mercadotecnia), The Bussines Policy Game (Administración General), Threshold (Administración General de Pymes), Fingame (Finanzas), Bussines Strategy Game (Estrategia, Administración General y Negocios Internacionales), entre otros aplicados a la ciencia administrativa, por ultimo cn las simulacioncs híbridas, se realiza la clase teórica y la práctica simultáneamente, con el desarrollo de casos prácticos van adelantando los temas teóricos del curso, algunos de los simuladores comerciales, citados anteriormente, incluyen los módulos de capacitación práctica con la teoría incluida, este tipo de simulación es muy utilizada en cursos y asignaturas de carácter virtual o cursos bajo la modalidad de créditos académicos donde dentro del proceso de formación es vital el trabajo independiente por parte de los estudiantes.

Scgún Emshoff y Sisson (1970), los modelos de simulación se clasifican según su naturaleza y sus características en: estáticos-dinámicos (dependiendo de lo estático o dinámico del problema o situación a representar), agregados o detallados (dependiendo del nivel de los objetos definidos dentro del modelo de simulación), físicos o de comportamiento (dependiendo de la inclusión de elementos físicos o características de comportamiento en el simulador que limiten la toma de decisiones), computador o humano (dependiendo de la influencia del ser humano dentro del proceso de simulación ${ }^{10}$ ), recursivos o de equilibrio (dependiendo de la complejidad de la situaciones ${ }^{11}$ ), , continuos o discretos (dependiendo del tipo de variables incluidas cn cl sistema), los modelos de tamaño o de escala (modelos sencillos de maquetas, tamaño real a menor o mayor escala, bidimensional, tridimensional, entre otros) y los modelos de simulación deterministicos o estocásticos (deterministicos, donde las variables endógenas y exógenas no se pueden tomar como datos al azar; estocásticos, donde por lo menos una variable es tomada como un dato al azar las relaciones entre variables se toman por medio de funciones probabilísticas, sirven por lo general para realizar grandes series de muestreos, requieren de bastante ticmpo en cl computador, son muy utilizados en investigaciones científicas).

10 En algunos simuladores se requiere poca interacción del individuo, en otros simuladores es imposible ejecutar la simulación sin la información que incluye el operador en el proceso de simulación.

11 En algunos se pueden llegar a situaciones de equilibrio, en otros casos siempre hay un único ganador, en otros casos ninguno de los agentes que participan en el proceso de simulación obtiene buenos resultados, todos pierden. 


\section{El proceso de la simulación}

El proceso de simulación se puede dividir en tres fases: la primcra fase, es la de desarrollo del modelo, la segunda fase, la de ejecución del modelo y la tercera fase, la de análisis de las salidas del modelo. Fn la primera fase, Desarrollo del Modelo, se siguen los siguientes pasos: 1) Formulación del problema, 2) Establecer los objetivos y plan general del proyecto, 3) Conceptualización del modelo, 4) Recolección de datos, 5) Implantación ${ }^{12}$ del modelo, 6) Si el modelo no se verifica, el proceso se regresa al punto 5 .

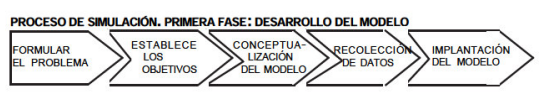

En la segunda fase, Ejecución del Modelo, se realiza de la siguiente forma: 1) Diseñar experimentos, 2) Experimentar con el modelo $\left.{ }^{13}, 3\right)$ Si el modelo tiene algún problema o no es valido se debe regresar a la primera fase, la fase de desarrollo del modelo.

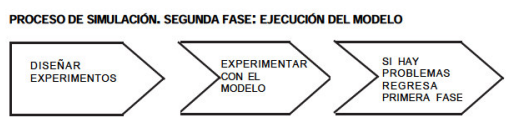

En la tercera fase, Análisis de Salidas del Modelo, los pasos a seguir son: 1) Analizar estadísticamente las salidas del modelo, 2) Si se necesitan mas ensayos o pruebas, se regresa a la fase de ejecución del modelo, 3) Si el modelo no es valido, deben regresar a desarrollo del modelo.

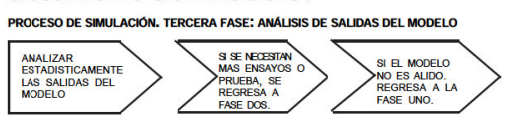

Fs muy importante analizar la diferencia entre los conceptos verificar y validar, un simulador ha sido verificado, cuando dentro de la primera fase, el paso 5) Implantación o programación corresponde al paso 3) Conceptualización del modelo, pero un simulador no es valido, cuando su comportamiento en la tercera fase, no funciona de acuerdo al paso de conceptualización en la primera fase.

Dentro de los elementos de un modelo de simulación, están incluidos: los procesos, los eventos, las actividades, los parámetros (constantes con valores exógenos), las variables de estado (recursos o elementos que pueden afectar las variables de estado que representan el estado del sistema simulado), las entidades o unidades de información y sus atributos o propiedades. Los eventos son acciones que ocurren a lo largo del funcionamiento de la simulación, las actividades son generadas periódicamente dentro de la simulación, el modelo de simulación internamente funciona en un tiempo

12 Existen procesos de simulación donde se requiera de programación, en otros procesos simplemente es una implementación o una adaptación de una solución que se parametriza a la medida de la organización o del problema.

13 Es necesario la realización de pruebas de ejecuciones para analizar el rendimiento técnico del simulador, la facilidad de operación, para realizar los ajustes necesarios antes de implementarlo. 
determinado. ${ }^{14}$

La principal de las ventajas que tienen los modelos de simulación, es que permiten realizar experimentos sin afectar directamente la realidad, facilitando observar sus efectos para apoyar la toma de decisiones, entre las desventajas que existen en los modelos de simulación están: el modelo no representa con exactitud la situación real, en el inicio del proceso de simulación se puede intervenir en la operación del sistema, los sistemas son operados por personas y por esta razón se generan problemas, en ocasiones todas las condiciones son continuas para el sistema siendo muy difícil en algunas simulaciones contar con el mismo tamaño de la muestra, en la mayoría de los simuladores es muy complicado tener en cuenta todas las alternativas o todas las variantes que pueden existir en la realidad, los modelos de simulación no generan todas las soluciones posibles ni las respuestas a algunas preguntas sobre el problema o la realidad simulada pero se convierten en una buena aproximación de la realidad, permitiendo la experimentación.

La simulación se puede utilizar: para la solución de problemas complejos, donde no se tenga un modelo matemático definido, cuando exista una formulación cxacta del sistema, al tcner fórmulas analíticas y se requiere un modelo para implementarlas, cuando la ejecución del modelo es más económico que intentar una solución con un experimento en la realidad, cuando es la única opción para generar las alternativas de solución a un problema, cuando se requicrc la manipulación del tiempo de los procesos dentro de un sistema, cuando se necesita por medio de la simulación hacer estudios y/ o experimentos que en la realidad no son posibles.

Es muy importante tener en cuenta que los modelos de simulación deben ser fáciles de entender y manipular por el usuario, deben tener claramente definidos las metas y los objetivos, las soluciones del modelo dependen de la información y las entradas suministradas en el proceso de simulación, deben permitir si se requiere, la parametrización del sistcma para adaptarlo a diferentes situaciones que pueden ocurrir en la realidad que representan, en proyectos de simulaciones complejas el desarrollo de los simuladores puede ser un proceso ejecutado en etapas, resolviendo uno a uno cada componente reduciendo la complejidad del problema.

\section{El impacto de la simulación}

En diferentes estudios como los publicados por James McKenney (1962), Anthony Raia (1966), Salim Jiwa (2005), Miles M. Smayling (2000), Jude Lee(1999), Brian H. Cameron (2003), Robert Cook (2004), Bill Davey (2005), entre otros, se ha planteado el impacto positivo y los limitantes de la utiliza-

14 El espacio no es un elemento fundamental de la simulación, en algunos casos se incluye como una variable o parámetro del modelo de simulación. 
ción de la simulación en los procesos de formación de profesionales en instituciones de educación superior o en procedimicntos de inducción y capacitación de personal en diferentes empresas, dentro de los procesos de formación en las funciones de organizar, planear, diseñar, dirigir o controlar en la administración de organizaciones, algunos en procesos específicos de toma de decisiones en situaciones complejas, otros en problemas de producción o en conceptualización y modelación de nuevos productos o servicios en organizaciones comerciales, comparando la actuación o desenvolvimiento de los individuos involucrados en estos procesos analizando si han desarrollado una formación solamentc tcórica, o si han utilizado solamente la practica por medio del simulador o en algunos casos si han utilizado los simuladores para desarrollar tanto el componente practico como para el conocimiento de la teoría, la gran mayoría de estos estudios, demuestran la validez de la utilización de la simulación y el gran impacto económico y operativo que ha tenido en estas situaciones, comparado en ocasiones los resultados obtenidos con los tres tipos de simulación existentes con el método tradicional de enseñanza sobre la realidad, estudios que involucraron las diferentes áreas de conocimiento incluyendo las administrativas. Entre las principales conclusiones se encuentran que los simuladores permiten la utilización de la teoría en situaciones complejas que pueden existir en la realidad, los simuladorcs aumentan la participación de los estudiantes y su motivación mas en nuestros días donde el uso de la tecnología por parte de los estudiantes es fundamental en su proceso de formación académico y profesional, por medio de los simuladores se mejora la transferencia de conocimiento y se aumenta la comprensión de los conceptos abstractos ${ }^{15}$.

En los últimos desarrollos de la simulación se incluyen los procesos de intercambio de información y de interacción con diferentes agentes en el proceso por medio de los simuladores en línea, que permiten al mismo tiempo la participación de varios operadores del simulador desde cualquier lugar del mundo por medio de Internet, donde cada uno puede manipular sus variables y parámetros, inclusive esta manipulación individual puede afectar los resultados a obtener por parte de los otros operadores, este tipo de simuladores, generan también actitudes de competencia en los participantes en su proceso de formación.

Algunos ejemplos de simuladores utilizados en los procesos de formación dentro de las ciencias administrativas, son : Markstrat, The Business Stratcgy Game y Bizplan Plan Builder, entre otros.

Markstrat ${ }^{16}$ es una herramienta muy efectiva para aprender estrategias

15 Un ejemplo sobre este impacto es el realizado por H W Lee(2005), con el desarrollo de una plataforma para gestión del conocimiento EPSS y la medición de su impacto.

16 Es posible visitar un demo del simulador en: http://www.dii.uchile.cl/ in58a01/ 
de marketing cuando se utiliza en conjunto con sesiones de clases teóricas. En particular, Markstrat aplica conceptos claves en cl discño de una cstratcgia de marketing como son: las estrategias de portafolio de producto, segmentación y posicionamiento al igual que el diseño de planes de marketing operativos. Este simulador es utilizado para desarrollar una masa crítica de tomadores de decisiones con orientación al mercado en todo tipo de organizaciones. El simulador permite al operador desarrollar habilidades para el diseño de estrategias en un entorno de riesgo. Al igual que un simulador de vuelo, Markstrat permite al operador practicar nuevas habilidades en un ticmpo limitado antes de aplicarlas en el mundo real. Los modelos matemáticos que están detrás de este simulador se basan en conceptos y teorías que han sido probadas durante muchos años por distintos tipos de organizaciones. En la simulación, Markstrat no pretende representar exactamente un mercado o una industria en particular, está desarrollada en un país ficticio de aproximadamente 250 millones de habitantes cuya unidad monetaria es el Markstrat Monetary Unit (MMU), simbolizada por \$. Este mundo Markstrat se comporta globalmente como la mayoría de los mercados, con todos los principios generales de marketing que se adquieren con la experiencia, la lectura de textos o la asistencia a clases. Fn la simulación los participantes forman equipos, cada uno administra una organización. En la operación se desarrollan estrategias a largo plazo con: la cartera de productos, el diseño de productos, la distribución , la fijación de precios, la publicidad y la fucrza de ventas. Se tomarán decisiones bajo incertidumbre sobre las condiciones del mercado, incluyendo las acciones que pueden realizar sus competidores.

The Business Strategy Game, es un simulador de negocios que permite a los participantes fabricar y vender productos en Norteamérica, Asia, Europa y Latinoamérica; además, cuenta con la opción de competir con otras empresas para ser el proveedor de una marca privada de calzado para una cadena norteamericana detallista. Cada empresa en The Business Strategy Gamc pucde optar por vender sus productos por medio de diferentes canales de distribución y cada equipo es responsable de idear estrategias con el objetivo de vencer a las empresas rivales. El juego está diseñado para que los participantes tomen decisiones en diferentes áreas funcionales de la empresa, lo cual ayuda a que las personas involucradas integren los conocimientos adquiridos durante los estudios o en la propia experiencia profesional. La misma dinámica del juego permite observar a la empresa como un todo, haciendo que el participante comprenda la importancia del pensamiento estratégico y su aplicación para proporcionar una ventaja competitiva frente a los contrincantes. No cabe duda que los participantes aprenderán sobre análisis financiero, toma de decisiones, trabajo en equipo y sobretodo aplicarán distintas opciones para 
tratar de alinear la estrategia corporativa con las estrategias de los departamentos de producción, mercadotecnia, finanzas y recursos humanos.

Bizplan Plan Builder $8^{17}$, un simulador que con sus ayudas le permite al usuario procesar sus ideas y recrear cada movimiento, con un sistema intuitivo, ingeniosamente diseñado, incorporando varios años de experiencia en cada industria, con diferentes clientes y con los soportes financieros específicos para cada sector. Este simulador ha sido desarrollado con mas de 600.000 clientes y millones dólares aportados a través de empresas capitalistas, de inversionistas, de los bancos y de la gerencia corporativa, en la simulación. El Bizplan Plan Builder 8, es la herramienta probada para desarrollar el concepto del negocio y presentarlo a los inversionistas que se puedan interesar, incluye un nuevo sistema con cinco diferentes tipos de planes de negocio que se pueden adaptar a las necesidades específicas de cualquier negocio, incluyendo: el plan del concepto, el plan del servicio, el plan al detal, el plan de producto, el plan del Internet, con tres niveles de modelos financieros, modelos que ayudan confirmar que incluye todo lo que requerido para desarrollar un plan de negocio.

Se pueden incluir dentro de los simuladores en línca, que permiten mayores niveles de colaboración e interacción desde cualquier lugar del mundo por medio de Internet ${ }^{18}$, al Simpro y Simdef, y estrategias de algunas Universidades o Asociaciones, que vinculan a diferentes participantes a procesos de simulación mediante concursos de competencia, uno de estas estrategias es la desarrollada por el Consejo Latinoamericano de Escuelas de Administración - CLADEA con el latin marketin game $^{19}$.

El Simpro ${ }^{20}$, donde los participantes toman la gerencia de una fábrica metalmecánica, programando la producción de 3 productos y la asignación de recursos humanos entre un grupo de 28 obreros con diferentes potenciales de productividad, las decisiones cubren un día de producción pero la demanda se surte cada tres días, la producción incluye 3 máquinas de preparación de productos, cada una de las cuales prepara los tres productos, y otras tres máquinas de acabado igualmente intercambiables. El problema de asignación no tiene solución óptima, el equipo ganador es aquel que logra el costo más

17 Para mayor información sobre el simulador puede visitar http://store.purplus.net/ biaplanbuil8.html

18 Son desarrollo de simuladores del LABSAG que es un ambiente virtual integral donde los participantes pueden tomar decisiones en 7 escenarios competitivos especializados, y en 2 escenarios integrales. Para mayor información sobre el simulador puede visitar http://www.gerentevirtual.com

19 Para mayor información sobre el simulador puede visitar http://www.cladea.org

20 Para mayor información sobre el simulador puede visitar de http://gerentevirtual.com/ escenarios.htm 
bajo de producción, dentro de sus objetivos están: 1) Acumular experiencia en toma de decisiones sobre control de inventarios y asignación de mano de obra a maquinaria. 2) Practicar en la aplicación de técnicas tales como: análisis incremental, programación de producción y modelos de lotes económicos. 3) Aprendizaje y dominio de conceptos y técnicas básicas en el manejo de operaciones. 4) Experiencia en la toma de decisiones interdependientes con un muy corto horizonte de tiempo pero con grandes consecuencias acumuladas a largo plazo.

El simulador Simdef ${ }^{21}$, es utilizado para fortalecer las habilidades de los participantes en contabilidad administrativa, producción y finanzas al requerirles una aplicación repetida de los principios, herramientas y procedimientos de esas disciplinas. En el caso de finanzas, la atención se concentrará en la toma de decisiones sobre la estructura financiera de la empresa y la asignación de recursos. Para obtener una operación fluida de la empresa el participante deberá pronosticar, planear y controlar. A través de Simdef se obtiene información sobre la interdependencia entre diferentes variables de decisión y puede construir una estructura de toma de decisiones sólidas para la cmpresa. Un conjunto de modclos financieros consistentes incorporados a Simdef permiten al participante construir un juego completo de decisiones financieras. Fsto se realiza dentro de un ambiente económico y con incertidumbre. Y por supuesto también con una dosis realista de riesgos. Tiene la capacidad de simular un mercado genćrico "commodity" muy cambiante dominado por la demanda donde las empresas no pueden influir en el precio. Minimizando la función de marketing, acrecentándose las de finanzas y producción, con grandes instalaciones de producción requieren enormes cantidades de capital depreciándose con rapidez, los participantes "compran" capital en un mercado de capitales sofisticado: préstamos bancarios, bolsa para acciones comunes y preferenciales, bonos, factoraje, valores negociables. Si no pueden hacerlo los prestamistas informalcs les facilitan dincro muy caro, se debe planearse a corto y largo plazo, seleccionar métodos de pronóstico y elegir entre proyectos de inversión.

Fistos son algunos de los simuladores existentes que apoyan la toma de decisiones en la administración de organizaciones y se constituyen en una herramienta importante en los procesos de formación de estudiantes y miembros de una organización.

\section{La simulación y el aprendizaje}

Es importantc realizar una reflexión sobre los cambios fundamentales en los procesos de aprendizaje y especialmente en la transformación de la relación profesor-alumno (Morales \&

21 Para mayor información sobre el simulador puede visitar http://gerentevirtual.com/ escenarios.htm 
Landa, 2004), algunos de estos cambios responden al desarrollo tecnológico y a los procesos de globalización quc ha vivido la humanidad, cn otros casos los cambios se realizan por reflexiones internas y nuevos movimientos científicos al interior de las diferentes disciplinas de conocimiento, en la mayoría de los casos aceptando la necesidad de la utilización de la practica en los procesos de formación (Garcia, Membrillo \& Cepeda, 2003).

Revisando los tipos de enfoques pedagógicos planteados por Luis Not en 1984, heteroestructuración y a u t o e s t r u c t u r a c i ó n; heteroestructuración, donde desde el exterior se forma al estudiante, con una cstructura de curso y temas que sc desarrollan con lecturas obligatorias, complementadas con otras lecturas recomendadas, y una posterior presentación al grupo por parte del profesor de cada tema con ejemplos y casos, o el enfoque de autoestructuración, donde se definen los objetivos del curso y cada estudiante construye casi un curso a su medida, teniendo en cuenta su nivel de conocimiento y los recursos bibliográficos y tecnológicos que tenga a su disposición, en este perspectiva se puede plantear utilizando la tipología de las simulaciones planteadas por Lce en 1999, que cl cnfoque de hetereoestructuracion se puede apoyar con simulaciones del tipo de presentación y simulaciones de práctica, mientras que el enfoque de autoestructuración se puede desarrollar $100 \%$ con la utilización de simulaciones híbridas, donde el estudiante en el desarrollo de su trabajo independiente o el trabajo dirigido dándole una cierta flexibilidad al enfoque, desarrolle su propio conocimicnto con su ritmo de trabajo.

Sin importar el enfoque pedagógico, la utilización de herramientas pedagógicas como la simulación es importante para mejorar los procesos de formación obteniendo mejores resultados en los estudiantes.

Dentro de las debilidades del método tradicional de enseñanza se encuentran: La dificultad que tiene el estudiante para la utilización de la teoría aprendida en la practica (la transformación del conocimiento en una habilidad), las limitantes en las relaciones humanas que ticne el cstudiantc debido a que su proceso de formación es en una sola vía la de recibir conocimiento, lo poco creativo que resulta un estudiante que no ha tenido la posibilidad de practicar o desarrollar su conocimiento adquirido, la evaluación de solamente la teoría transmitida no garantiza su aprendizaje y su correcta utilización, esto limita la formación integral del individuo que al terminar su proceso de formación se debe integrar en un sistema social de producción donde interactúa con otros individuos en la solución de problemas, solamentc con cl conocimicnto transmitido de forma unilateral. (Florez, 1994)

La metodología de estudios de casos y enseñanza basada en la experiencia, traen consigo una serie de ventajas como: permiten la interactividad y la colaboración, se analizan problemas más complejos, existen diferentes 
métodos para realizar el análisis a un problema desde varias perspectivas, permiten el aprendizaje activo, en un campo de acción mas amplio que cl método tradicional, algunas de estas ventajas son planteadas por Dave (2005), Obrien (2002), entre otros, y conviertiendose en los principios metodológicos para el desarrollo de la simulación.

La utilización de los simuladores en los procesos de formación desarrollan destrezas y habilidades en los individuos fundamentales para su practica profesional, aumentando la posibilidad de lograr mejores resultados en la toma de decisiones o en la solución de problemas dentro de una organización, gencrando una mayor confianza a las organizaciones que analizan las competencias profesionales y personales de los individuos a vincular a su organización.

\section{La simulación y las competencias profesionales}

La globalización de la economía, la competitividad de las organizaciones y la evolución de los sistemas productivos, han requerido de una trasformación en los procesos de selección y desarrollo profesional de los individuos a vincular o vinculados en las organizaciones. Pensando la organización como un sistema donde cada elemento debe generar una sinergia con los otros para el cumplimiento de los objetivos organizacionales como sistema ${ }^{22}$, cste nucvo clemento del sistema debe tener unos conocimientos y destrezas que le garanticen a la organización su correcto funcionamiento dentro del sistema.

Para Arguelles (2002), estos conocimientos y destrezas deberían responder a un proceso de formación de cada individuo, que se dirigida al desarrollo de conocimientos técnico-científicos y culturales, incluyendo conductas y actitudes básicas de carácter personal que no son el resultado del proceso de formación dentro de una institución, sino de su compromiso como ser social, en ocasiones el desarrollo de estas actitudes y conductas son incluidas en el currículo de un programa académico para garantizar su comprensión y conocimiento. Según Mertens (2000), dentro de estas características están: la capacidad de resolver problemas (habilidad para enfrentarse y dar solución a una situación determinada), la capacidad de organización del trabajo (habilidad para utilizar los recursos humanos o materiales de forma eficiente), la capacidad de responsabilidad en el trabajo, la capacidad de trabajar en equipo y de relación interpcrsonal (habilidad para colaborar dentro de un equipo de personas para lograr un objetivo), la autonomía (capacidad de realizar una tarea

22 Se refiere a cada nuevo trabajador o colaborador de una organización, que se integra al sistema y debe generar sinergia dentro del sistema que se comporta como una totalidad. 
de forma independiente), la capacidad de iniciativa (habilidad para tomar decisiones) y la capacidad de innovación (habilidad para mcjorar un proccso productivo), entre otras competencias de carácter personal, que se pueden desarrollar en los procesos de formación en una institución de educación o en los procesos de inducción dentro de una organización por medio de la utilización de simuladores, donde el individuo aprende al enfrentarse a diferentes situaciones simuladas, realizando una medición de su actuación y de sus resultados, incluso permitiéndole al individuo conocer la correcta forma de actuar para la resolución del problema o ante una situación especifica $^{23}$, cstas capacidades o habilidades no se pueden desarrollar con los modelos tradicionales de transmisión de conocimiento, requieren para su desarrollo de la practica, al desarrollarse por medio de la simulación puede además de ser mas amplia, generar un mayor impacto en la formación de los individuos tanto en las competencias laborales técnicas como en las competencias laborales personales.

\section{Conclusiones}

La complejidad de las organizaciones vista como sistemas puede ser reducida, permitiendo su análisis y su comprensión, facilitando la resolución de problemas y la toma de decisiones. Fstos sistemas complejos reducidos, pueden ser llevados a procesos de construcción de modelos de simulación, por medio de los cuales se permitc su estudio en cl presente y la realización de predicciones sobre su futuro. La simulación se ha convertido en una herramienta para reducir la complejidad y el análisis de algunos fenómenos organizacionales.

Desde sus orígenes la simulación se constituyó en una herramienta que cambio los métodos de enseñanza tradicionales y generó grandes aportes a las organizaciones, en nuestros días los simuladores siguen evolucionando de forma técnica llegando a simuladores en red desarrollados lenguajes de programación de cuarta generación, como de forma opcrativa solucionando problemas cada día mas complejos con un mayor numero de variables y agentes, disminuyendo los altos costos en ocasiones que generan los procesos de toma de decisiones, reduciendo en un alto grado los niveles de incertidumbre.

En los métodos de enseñanza se han realizado diferentes estudios sobre el impacto en el aprendizaje de la utilización de la simulación como una herramienta pedagógica, confirmando el gran aporte que generan en el desarrollo de las competencias laborales tćcnicas y personales.

Para las ciencias organizacionales la simulación permite la creación de laboratorios para experimentar, disminuyendo los costos y los tiempos de

\footnotetext{
Un buen ejemplo en el campo de la economía, es la utilización de la simulación en estudios macroeconómicos, planteado por Woltjer (2005).
} 
la realización de pruebas en la realidad, con la evolución de los simuladores se reduce la brecha entre la virtualidad y rcalidad.

Cada vez se desarrollan mejores simuladores con tecnologías como la realidad virtual y la inteligencia artificial, los procesos de simulación son más reales, con un mayor impacto en la solución de problemas o los estudios sobre el futuro, generando un gran beneficio para la administración de organizaciones.

Dentro de estos desarrollos de la simulación es fundamental la relación Universidad-Empresa, este vinculo es vital, la empresa le brinda a la Universidad, la información de casos reales que experimenta día a día, la Universidad incluye el conocimiento teórico para la solución de problemas, y por medio del proceso de la simulación se incluyen en el resultado final que es el simulador. Con el simulador la organización, puede antes de la toma de una decisión simular su efecto y de esta forma tomar la mejor decisión como resultado de un proceso científico.

Es muy importante para las organizaciones la utilización de la simulación en el desarrollo de las competencias profesionales y personales de sus miembros, incluyendo los nuevos micmbros a vincular, con los simuladores existentes es posible lograr este objetivo, pero aunque la mayoría de los simuladores permiten adaptaciones al medio donde se requiera su implementación, hay variables y parámetros específicos que posiblemente no se puedan incluir en los pro- cesos de simulación utilizando estas herramientas, no logrando una recreación exacta de la realidad, o una mejor aproximación a la misma, por csto se hace necesario iniciar procesos de desarrollo de simuladores en Universidades y centros de investigación, que respondan al análisis de la realidad del medio que nos rodea, este desarrollo de nuevos simuladores debe ser un proceso interdisciplinario, donde intervengan expertos de cada disciplina en la construcción del modelo, definiendo las variables y parámetros propios del sistema y del entorno, es fundamental la participación de diferentes organizaciones para por medio de su análisis y con su información, parametrizar y desarrollar cada simulador, es fundamental realizar las diferentes etapas para el desarrollo de los simuladores donde son muy importantes las pruebas que se realicen de su operación y funcionamiento, su implementación debe realizarse en plataformas compartidas para ser utilizadas por instituciones de educación y por organizaciones de todo tipo, permitiendo la toma de decisiones y solución de problemas en nuestras organizaciones incluyendo la mayoría de variables propias del entorno logrando un mejor resultado.

\section{Bibliografia}

Arguelles, A (2002). Competencia laboral y educación basada en normas de competencia. Mexico, Limusa.

Bertalanffy, L (1984). Teoría General de Sistemas. Fondo de Cultura Económi- 
ca.

Bertalanffy, L (1986). Perspectivas de la Teoría General de Sistemas: Estudios científico-filosoficos España. Alianza Editorial.

Brend, D (1999). El aprendizaje basado en la experiencia: la búsqueda de un nuevo paradigma para la enseñanza y el aprendizaje. Simulation \& Gaming Vol. 30 No. 4

Burch, J (1969). Business Games and Simulation Techniques. Management Accountig 51,6.ABI/INFORM GLO$B A L$.

Cameron, B (2003). Effectiveness of Simulation in a hybrid and online networking course. The Quarterly Review of Disgtance Education, Vol 4 No.1

Cook, R (2004). The Pedagogy and Efficay of Using a Sales Management Simulation: The MARS Sales Management Simulation Experience. The National Conference in Sales Management.

Cornejo, A (2004). Complejidad Organizacional. Complejidad y Caos. Guia para la administración del siglo $X X I$.

Davey, Bill (2005). System analysis education using simulated case studies. Issues in informing science and information technology.

Descartes, R (1974). Discurso del Método.10ed, Argentina, Losada.

Dewey, J(1949). Experience and Existence: A comment. Philosophy and Phenomenological Research, 9, 4.

Dewey, J (1906). Reality as Experience. Journal of Philosophy, Psychology and scientific methods, 3,10.

Emshoff, J \& Sisson, R (1970). Design and use of computer simulation models. Macmillan publishing Co.

Faria, A. (1990). Business Simulation games after thirty years: Current usage levels in the United States. Guide to Business Gaming and Experiential Learning. Cap. 4.

García, C, Membrillo, A \& Cepeda J.(2003) Una experiencia de aprendizaje: Modelo y Simulación de Decisiones Estratégicas de Negocios. 1er Congreso de Dinámica de Sistemas Latinoamérica, Monterrey, México.

Hall, R (1982). Organizaciones estructura y proceso. $2 \mathrm{Ed}$, Prentice Hall.

Jiwa, S. (2005). E-Entrepreneurship: Learning in a simulated environment. Journal of electronic commerce in organizations, 3.

Johansen, O (1992). Introducción a la Teoría General de Sistemas. Grupo Noriega Editores.

Katz,D \& Kahn, R (1979). Psicología social de las organizaciones. Editorial Trillas.

Klir,G \& Bertalanffy, L. (1987). Tendencias en la teoría general de sistemas. Alianza. Editorial.

Kuhn, T(1980). La estructura de las revoluciones científicas. Fondo de Cultura Economica.

Lee, H. (2005). Contemporary Knowledge Management Plataform - EPSS. Journal of American Academy of Business. Cambrige. 7,1. ABI/INFORM GLOBAL

Lee, J (1999). Effectiveness of Computerbased Instructional Simulation: A Meta Analysis. International Journal of Instructional Media, 26.

Luhmann, N. (1995). Poder. Editorial Anthropos.

Luhmann, N. (1996). Confianza. Editorial Anthropos.

Luhmann, N. (1997). Sociedad y Sistema: La ambición de la teoría. Ediciones paidos ibérica S.A.

Mertens, L (2000). ISO 9000 y compe- 
tencia laboral. E1 aseguramiento del aprendizaje continuo en la organización. FORO MUNDIAL INLAC.

Morales, P y Landa V. (2004). Aprendizaje basado en problemas - problem based learning. En Theoria, 13.

Morgan, G. (1998). Imágenes de la Organización. Alfaomega.

Morin, E (1991). El Método III : El cono cimiento del conocimiento. Catedra.

McKenney J (1962). An evaluation of a Business Game in an MBA Curriculo, Management Technology, $3,1 . i$

Negroponte, N (1995). Ser Digital. Atlantida.

Not, Luis. (1984). La pedagogía del conocimiento. FC.

O’Brien (2002). A response to "Dewey and Vygotsky: Society, Experience, and Inquiry in Edutational Practice. Educational Researcher, 3, 5.

Ochoa R (1994). Hacia una pedagogía del conocimiento. Mcgraw Hill.

Popper K, Adorno T, Dahrendorf R \& Habermans J (1969) La Lógica de la Ciencias Sociales. Grijalbo.

Raia A (1966). A Study of the Educational Value of Management Games. Journal of Business, 39, 3.

Ross, W. D. (1981) Aristoteles. 2Ed, Editorial Charcas.

Samuel, E (1963) Management Games. Operation Research Society, 14, 2.

Schneier, C. (1977) Experiential learning: Toward the development or a theoretical base and the identification of variables and hypotheses to guide research. New Horizon in Simulation Games and Experiential Learning.4.

Smayling M.(2000). A comparison of student performance on a business simulation and otherrequirements. Journal of Business Education, 1.

Úbeda, M \& Llopis, F. (2002). Organizational learning in a global market. Human Systems Management 21. IOS Press.

Wolfre, J (1976). The Effects and Effectiveness of Simulations in Business Policy Teaching Applications. The Academy of Management Review, 1, 2.

Woltjer, G (2005). Decisions and Macroeconomics: Development and Implementation of a Simulation Game. Journal of Economic Education. 36, 2 ABI/INFORM GLOBAL 